General Letters in Mathematics (GLM) I(1) (2016) 39-44
General Letters in Mathematics (GLM)
Website: http://www.sciencereflection.com/general-letters-in-mathematics/
Reflection

\title{
Existence and Regularity Results for the Stokes System with Mixed Boundary Conditions
}

\author{
Mourad Dilmi $^{1}$, Hamid. Benseridi ${ }^{2}$ \\ 1,2 Applied Math Lab, Department of Mathematics, Setif I-University, 19000, Algeria. \\ 1 dilmorad@gmail.com
}

\begin{abstract}
In this paper we consider the nonlinear boundary value problem governed by a stationary perturbed Stokes system with mixed boundary conditions ( Dirichlet-Fourier- maximal monotone graph), in a smooth domain. The existence and regularity of the weak solution to this problem are proved. Our approach is based on the maximal monotone graph by its Yosida regularization and the contraction method of Brezis [3].
\end{abstract}

\section{Indexing terms/Keywords}

A priori inequalities; maximal monotone graph; Stokes; Variational problem.

\section{SUBJECT CLASSIFICATION}

MSC[2010]: . 35B40, 35B65, 35C20.

\section{Introduction}

If $S$ is Many research papers have been written recently, both on the existence, uniqueness and regularity of solutions of Stokes system in different domains but with the usual boundary conditions (Dirichlet, Neumann, ...), see for example $[1,3,5,9, \ldots]$ and the references cited therein. In [2] the regularity of a stationary equation for a non-isothermal Newtonian and incompressible fluids, in a three-dimensional bounded domain is studied. The problem is governed by a coupled system involving a balance of linear momentum and the heat energy with Tresca free boundary friction conditions.

This work is devoted to the study of the existence and regularity for the solution of the following problem. Let $\Omega$ be a bounded open subset of $\mathrm{R}^{n}(n=2,3)$ of class $C^{2}$. The boundary $\Gamma=\partial \Omega$ is assumed to be composed of three parts: $\bar{\Gamma}_{1}, \bar{\Gamma}_{2}$ and $\Gamma_{3}$ with measure $\left(\Gamma_{1}\right)>0$. The notation $\beta$ will stand for a 
maximal monotone graph such that $0 \in \beta(0)$. For given body forces $f \in L^{2}(\Omega)^{n}$, we look for a solution $(u, p)$ in $H^{2}(\Omega)^{n} \times H^{1}(\Omega)$ of the following problem:

$$
\left\{\begin{array}{ccc}
-v \Delta u+k^{2} u+\nabla p=f & \text { in } & (\Omega) \\
\operatorname{div}(u)=0 & \text { in } & (\Omega) \\
u=0 & \text { on } & \left(\Gamma_{1}\right) \\
\sigma_{\tau}(u)+\alpha u=0, \\
u \cdot n=0 ; \\
-\sigma(u) \cdot n+L(u) \in \beta(u) & \text { on } & \left(\Gamma_{3}\right)
\end{array}\right.
$$

where $p, u, n$ and $v$ are ,respectively, the pressure, the velocity field, the unit outword normal to $\Gamma$ and the viscosity. $L$ a first order differential operator with libschitzian coefficients, $k$ is a real number to be fixed lateron. We recall that the components of the stress tensor itself are

$$
\sigma_{i j}(u)=-p \delta_{i j}+2 v \varepsilon_{i j}(u), \varepsilon_{i j}(u)=\frac{1}{2}\left(\frac{\partial u_{i}}{\partial x_{j}}+\frac{\partial u_{j}}{\partial x_{i}}\right) \quad(1 \leq i, j \leq n) .
$$

The formulation of boundary conditions, with maximal monotone graphs, involves several types of conditions resulting from physical problems, such as the Dirichlet, Neumann or Signorini conditions (see, [7]), a boundary condition involved in elasticity with friction in a problem of air conditioning (see, [6]).

The paper is organized as follows: In section 2 we give some preliminaries which will be needed below, while in section 3 we introduce a non-decreasing function $\beta_{\lambda}$ which is regularized in the sense of Yosida. Then we obtain a new nonlinear problem whose the fixed point method is not well adapted. We introduce an intermediate problem for which the Banach fixed point theorem holds. Finally, the a priori estimate allows us to pass to the limit when $\lambda$ tends to zero, we prove our main results of existence and regularity of the solution to our initial problem (1).

\section{Preliminary}

We In this paragraph we give some results of [10] which will be useful in the next.

Lemma 2.1. Let $L$ a tangent operator of the first order, for all $\phi \in L^{1}(\Gamma)^{n}$ such that $L(\phi) \in L(\phi) \in$ $L^{1}(\Gamma)^{n}$ we get:

$$
\left|\int_{\Gamma} L(\phi) d s\right| \leq c_{1} \int_{\Gamma}|\phi| d s
$$

where $c_{1}=c_{1}(\Omega, L)$ is a constant. 
Theorem 2.1. For $\Omega$ of class $C^{0,1}$ and for any $\gamma>0$, there exists a constant $c_{2}(\gamma)$ depending only on $\gamma$ such that:

$$
\begin{gathered}
\|\varphi\|_{L^{2}(\Gamma)^{n}}^{2} \leq \gamma\|\nabla \varphi\|_{L^{2}(\Omega)^{n \times n}}^{2}+c_{2}(\gamma)\|\varphi\|_{L^{2}(\Omega)^{n}}^{2}, \forall \varphi \in H^{1}(\Omega)^{n} . \\
\|\nabla \varphi\|_{L^{2}(\Gamma)^{n \times n}}^{2} \leq \gamma\|\varphi\|_{H^{2}(\Omega)^{n}}^{2}+c_{2}(\gamma)\|\varphi\|_{H^{1}(\Omega)^{n}}^{2}, \forall \varphi \in H^{2}(\Omega)^{n} .
\end{gathered}
$$

\section{Main Results}

To reach the desired goal, let us introduce non-decreasing functions $\beta_{\lambda}$ which are regularized in the sense of Yosida of $\beta$ defined by: $\beta_{\lambda}=\lambda^{-1}\left(I+J_{\lambda}\right)$, where $J_{\lambda}=-(I+\lambda \beta)^{-1}$ is the resolvante of $\beta$.

At first time, we consider the following approached problem:

$$
\left\{\begin{array}{ccc}
-v \Delta u_{\lambda}+k^{2} u_{\lambda}+\nabla p=f & \text { in } & (\Omega) \\
\operatorname{div}\left(u_{\lambda}\right)=0 & \text { in } & (\Omega) \\
u_{\lambda}=0 & \text { on } & \left(\Gamma_{1}\right) \\
\sigma_{\tau}\left(u_{\lambda}\right)+\alpha u_{\lambda}=0, \\
u_{\lambda} \cdot n=0 ; \\
\left(L u_{\lambda}-\sigma\left(u_{\lambda}\right) n\right)=\beta_{\lambda}\left(u_{\lambda}\right) & \text { on } & \left(\Gamma_{3}\right)
\end{array}\right.
$$

The nonlinear problem (3.1) is not well adapted to the fixed point method, an other difficulty is to give the priori estimate, so we introduce the following intermediate problem for wich the Banach's fixed point theorem holds.

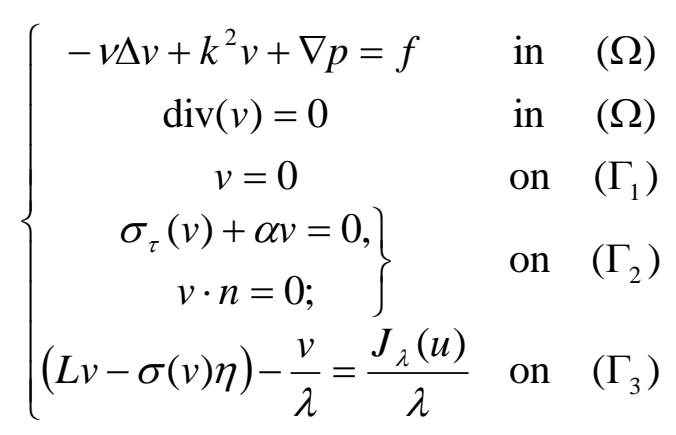

where $u \in H^{\frac{1}{2}}\left(\Gamma_{3}\right)^{n}$.

It is clear that each fixed point of (3.2) (i.e. a solution $v$ is found as $v_{\Gamma_{\Gamma_{3}}}=u$ ) gives a solution of (3.1). 


\subsection{Study of the intermediate problem (3.2)}

To get a weak formulation, we introduce

$$
\begin{aligned}
V_{\mathrm{div}} & =\left\{\varphi \in H^{1}(\Omega)^{n}: \varphi_{/_{\Gamma_{1}}}=0, \phi \cdot n=0 \text { on } \Gamma_{2} \text { and } \operatorname{div}(\varphi)=0\right\} ; \\
L_{0}^{2}(\Omega) & =\left\{q \in L^{2}(\Omega): \int_{\Omega} q d x=0\right\} .
\end{aligned}
$$

Theorem 3.1. There exists a unique $v \in V_{\text {div }}$ and $p \in L_{0}^{2}(\Omega)$ (up to an additive constant) solution to problem (3.2).

Proof. A formal application of Green's formula, using (3.2) leads to the weak formulation:

Find $v \in V_{\text {div }}$ such that: $a(v, \phi)=l(\phi), \forall \phi \in V_{\text {div }}$

where

$$
\begin{aligned}
a(v, \varphi) & =2 v \int_{\Omega} \varepsilon(v) \varepsilon(\varphi) d x+k^{2} \int_{\Omega} v \cdot \varphi d x+\alpha \int_{\Gamma_{2}} \omega \cdot \phi d s-\int_{\Gamma_{3}} L v \cdot \varphi d s+\frac{1}{\lambda} \int_{\Gamma_{3}} v \cdot \varphi d s \\
l(\varphi) & =\int_{\Omega} f \cdot \varphi d x-\frac{1}{\lambda} \int_{\Gamma_{3}} J_{\lambda}(u) . \varphi d s .
\end{aligned}
$$

Using (2.1)-(2.3) and the Korn's inequality (with its constant $c_{3}$ ). Choosing $\gamma$ and $k$ such that:

$$
\gamma<\frac{2 v c_{3}}{c_{1}}, \quad k \geq \sqrt{c_{1} c_{2}(\gamma)} \quad \text { and } \quad \alpha=\min \left(\left(2 v c_{3}-c_{1} \gamma\right),\left(k^{2}-c_{1} c_{2}(\gamma)\right)\right)
$$

we obtain that the bilinear form $\breve{a}(.,$.$) is continuous and coercive.$

The form $l$ is linear and continuous.

By the Lax-Milgram theorem, there exists a unique solution $v \in V_{\text {div }}$ of $a(v, \phi)=l(\phi), \quad \forall \phi \in V_{\text {div }}$; then the existence of $p$ is obtained by using a duality results of convex optimization.

Therefore, there exists $(v, p) \in V_{\text {div }} \times L_{0}^{2}(\Omega)$ solution of the problem (3.2).

Now we establish the solution of a nonlinear problem (3.1).

Theorem 3.2. Under the assumption of (3.4), there exists a unique $u_{\lambda} \in V_{\mathrm{div}}$, and a unique (up to an additive constant) $p \in L_{0}^{2}(\Omega)$, solution to the problem (3.1).

Proof. We use the Banach fixed point theorem. For this, we introduce the mapping defined by 


$$
\begin{gathered}
\Lambda: L^{2}\left(\Gamma_{3}\right)^{n} \rightarrow L^{2}\left(\Gamma_{3}\right)^{n} \\
u \rightarrow \Lambda(u)=v_{/ \Gamma_{3}},
\end{gathered}
$$

where $v$ is the solution of (3.2).

The mapping $\Lambda$ is strictly contracting, then there exists one and only one element $u \in L^{2}\left(\Gamma_{3}\right)^{n}$ such that $\Lambda(u)=u=v_{\Gamma_{\Gamma_{3}}}$ and $v$ is solution of (3.2). Finally, we have proved that the solution $\left(u_{\lambda}, p\right)$ of the problem (3.1) is in $V_{\text {div }} \times L_{0}^{2}(\Omega)$.

Theorem 3.3. If $k$ verify (3.4), the solution $\left(u_{\lambda}, p\right)$ of the nonlinear problem (3.1) satisfies $u_{\lambda} \in H^{2}(\Omega)^{n}$ and $p \in H^{1}(\Omega)$ (up to an additive constant).

\section{A priori estimate}

Let $\left(u_{\lambda}, p\right)$ solution of (3.1) we have the:

Theorem 4.1. Under the assumption of theorem 3.2 there exists a constant $C$ independent of $\lambda$ such that

$$
\left\|u_{\lambda}\right\|_{H^{2}(\Omega)^{n}}^{2}+\|\nabla p\|_{L^{2}(\Omega)^{n}} \leq C\|f\|_{L^{2}(\Omega)^{n}} .
$$

Theorem 4.2. If the inequality (3.4) holds, there exists a unique $u_{\lambda} \in H^{2}(\Omega)^{n}$, and a unique (up to an additive constant) $p \in H^{1}(\Omega)$, solution to the initial problem (1.1).

Proof. According to (4.1) there exists a sequence $\lambda_{j}$ tending to 0 such that

$$
\begin{array}{cc}
u_{\lambda_{j}} \rightarrow u & \text { weakly in } H^{2}(\Omega)^{n}, \\
u_{\lambda_{j}} \rightarrow u & \text { strongly in } H^{1}(\Omega)^{n}, \\
\operatorname{div}\left(u_{\lambda_{j}}\right) \rightarrow 0 & \text { strongly in } L^{2}(\Omega)^{n}, \\
\left.\begin{array}{c}
u=0 \\
\sigma_{\tau}(u)+\alpha u=0, \\
u \cdot n=0 ;
\end{array}\right\} & \text { on } \Gamma_{1}, \\
& \text { on } \Gamma_{2} .
\end{array}
$$

On the other hand

$$
\begin{gathered}
L\left(u_{\lambda_{j}}\right) \rightarrow L(u) \quad \text { in } L^{2}\left(\Gamma_{3}\right)^{n}, \\
\sigma\left(u_{\lambda_{j}}\right) \eta \rightarrow \sigma(u) \eta
\end{gathered}
$$

Thus $\beta_{\lambda_{j}}\left(u_{\lambda_{j}}\right) \rightarrow-\sigma(u) \eta+L(u)$ strongly in $L^{2}\left(\Gamma_{3}{ }^{n}\right.$. 
But since $\beta_{\lambda_{j}} \subset \beta \circ\left(-J_{\lambda}\right)$, we deduce that

$$
u_{\lambda_{j}}+J_{\lambda_{j}}\left(u_{\lambda_{j}}\right)=\lambda_{j} \beta_{\lambda_{j}}\left(u_{\lambda_{j}}\right) \underset{\lambda_{j} \rightarrow 0}{\rightarrow} 0
$$

which is equivalent to $-J_{\lambda_{j}}\left(u_{\lambda_{j}}\right) \underset{\lambda_{j} \rightarrow 0}{\rightarrow} u$.

Hence the limit $u$ satisfies (1.1) and then we have the regularity.

\section{References}

[1] Amrouche. C \& Girault. V, (1991). On the existence and regularity of the solution of Stokes problem in arbitrary dimension, Proc. Japan Acard 67, p 171-175.

[2] A. Ben-Israel and T. N. E. Greville, Generalized inverses, theory and applications, Springer-Verlag NewYork, Inc (2003).

[3] Brezis. H. Opérateurs maximaux monotones et semi-groupes de contractions dans les espaces de Hilbert, Mathematic Studies, North-Holland, (1973).

[4] S. R. Caradus, Generalized inverses and operator theory, Queen's paper in pure and applied mathematics, Queen's University, Kingston, Ontario,(1978).

[5] Cattabriga. L, (1961). Su un problema al contorno relativo al sistema di equazioni di Stokes, Rendiconti del Matematico della Università di Padova 31, p 308-340.

[6] Dauge, M. Etude de l'opérateur de Stokes dans un polygone: Régularité, Singularité et Théorèmes d'indices, Thèse de Doctorat de 3 ème cycle, Université de Nantes, (1980).

[7] Duvaut. G \& Lions. L, (1969). Nouvelles inéquations variationnelles rencontrées en thermique et en thermoélasticité, C.R.A.S, Paris, 269.

[8] Fichera. G, (1964). Problemi elastostatici con vincoli unilateral : problema di Signorini, con ambigue conditiozioni al contorno, Mem. Accad. Naz. Lincei Ser 8, 7.

[9] S. Izumino, The product of operators with closed range and an extension of the reverse order law, Tohoku Math. J. 34 (1982) 43-52.

[10] Necas. J. Les méthodes directes en théorie des équations elliptiques, Masson. Paris, (1967). M.Z. Nashed, Generalized Inverses, Theory and Applications, Academic Press, NY (1976).

[11] Saito. N, (2004). On the Stokes equations with the leak and slip boundary conditions of friction type: regularity solutions, Publications of RIMS, Kyoto University 40 345-383. 